\title{
Fermented Noni Exudate (fNE): A mediator between immune system and anti-tumor activity
}

\author{
JINHUA LI ${ }^{1}$, SARA L. STICKEL ${ }^{1}$, HILARY BOUTON-VERVILLE ${ }^{1}$, KELLY E. BURGIN ${ }^{1}$, \\ XIANZHONG YU ${ }^{1,2}$, DESMOND K.W. WONG ${ }^{3}$, THOMAS E. WAGNER ${ }^{1,2}$ and YANZHANG WEI ${ }^{1,2}$ \\ ${ }^{1}$ Oncology Research Institute of Greenville Hospital System University Medical Center, Greenville, \\ ${ }^{2}$ Department of Biological Sciences of Clemson University, Clemson, SC, ${ }^{3}$ Department of Alternative and \\ Complementary Medicine in the John Burns School of Medicine, University of Hawaii, Manoa, HI, USA
}

Received June 25, 2008; Accepted September 2, 2008

DOI: 10.3892/or_00000172

\begin{abstract}
The anti-tumor activity of Morinda citrifolia fruit juice (Noni) has been previously reported. However, the mechanism behind this activity remains unknown. In the present study, we studied the anti-tumor activity of fermented Noni exudate (fNE) and demonstrated that intraperitoneal injection of this material significantly increased the percentages of granulocytes and NK cells in the peripheral blood, peritoneum, and spleen. Furthermore, in preventive and treatment settings, fNE injection induced complete tumor rejection in normal C57BL/6J mice, partial tumor rejection in C57 nude mice lacking functional lymphocytes, and no tumor rejection in NK cell deficient beige mice. Over $85 \%$ of the C57BL/6J mice that received $\mathrm{fNE}$ survived the first tumor injection and rejected up to $5 \times 10^{6}$ tumor cells when re-challenged. The anti-tumor activity remains in the heat-inactivated and filtrated supernatant of fNE. These data demonstrate that fNE appears to be able to stimulate the innate immune system and the adaptive immune system to reject tumor cells. NK cells respond quickly and appear to be among the major players of the innate immune system, while the adaptive immune system reacts later with a retained memory.
\end{abstract}

\section{Introduction}

Morinda citrifolia (Noni) has been extensively used in folk medicine by Polynesians for over 2000 years (1-3). Recent studies have shown that Noni has a wide spectrum of therapeutic activities including inhibition of angiogenic initiation (4) anti-inflammatory effects (5), and anti-cancer activities. Liu et al (6) demonstrated that two glycosides from Noni inhibit

Correspondence to: Dr Yanzhang Wei, Oncology Research Institute, 900 W. Faris Road, Greenville, SC 29605, USA

E-mail: ywei@ghs.org

Key words: fermented noni exudate, S180, NK cells, innate immunity
AP-1 transactivation and cell transformation. Animal studies showed that Noni also has anti-tumor activity in vivo in various animal models (7-9). Wong has reported that two cancer patients have benefited by drinking Noni (10). However, the mechanism behind these observed anti-tumor activities remains unknown. It has been suggested that the prevention of carcinogen-DNA adduct formation and the anti-oxidant activity of Noni may contribute to its cancer preventive activity (8). Enhancement of anti-tumor immunity has also been suggested as a potential anti-cancer mechanism for the products of this plant $(7,9)$.

In this study, we studied the anti-tumor effect of $\mathrm{fNE}$ in a sarcoma 180 ascites model and other tumors and found that the innate and adaptive immune systems are involved in the antitumor activity. The innate immune system, especially NK cells, reacts quickly while the adaptive immune system appears to be required to develop anti-tumor memory.

\section{Materials and methods}

Mice and tumor cells. Female C57BL/6J, Nu/B6 nude, and beige $\mathrm{KO}$ mice $(\mathrm{C} 57 \mathrm{BL} / 6 \mathrm{~J}-\mathrm{Lyst}<\mathrm{bg}-\mathrm{J} / \mathrm{J})$ at ages 6-8 weeks were purchased from Jackson Laboratories and housed in our pathogen-free animal facilities. The animal experiments were carried out in accordance with the Guide-lines for the Care and Use of Laboratory Animals (NIH Publication Number 85-23) and the institutional guidelines. Mouse sarcoma S180 tumor cells (TIB-66) and LL/2 Lewis lung carcinoma tumor cells (CRL-1642) were purchased from ATCC and cultured in DMEM with $10 \% \mathrm{FBS}$ and gentamicin $(50 \mu \mathrm{g} / \mathrm{ml})$.

fNE production. Very ripe Noni (Morinda citrifolia) fruits were collected from Kawaihae on Hawaii's South Kohala coast. The fruit was still hard but almost completely whitish yellow in color. All processing equipment were cleaned and sterilized by UV light for at least $4 \mathrm{~h}$ and the fruit processed in a room with a humidity control between 50 and $60 \%$. The stems were removed to allow thorough cleaning with a diluted solution of dish washing liquid in tap water using a soft brush. They were then rinsed with running tap water. A second rinse was then done with deionized water. The fruits were then placed in clean and dry stainless steel colanders to drain off the 


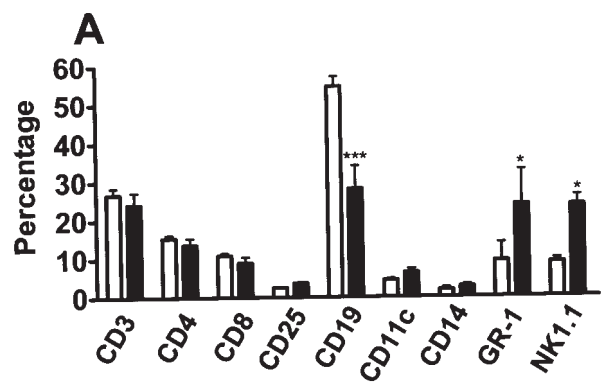

B
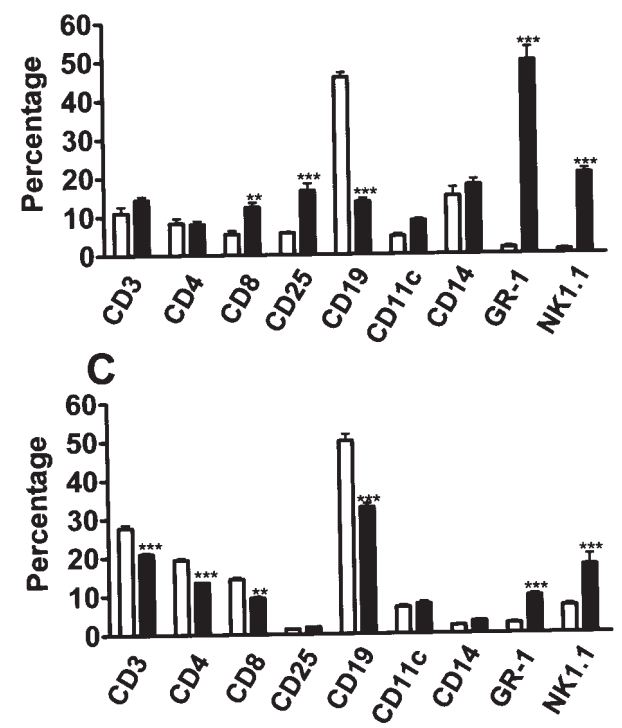

Figure 1. CD profiles of cells from peripheral blood (A), peritoneum (B), and spleen (C). C57BL/6J mice were i.p. injected with three doses of PBS (500 $\mu 1 /$ mouse/day, open bars) or three doses of fNE (500 $\mu 1 /$ mouse/day, solid bars). A day later, the mice were sacrificed and cells were collected from the three tissues. After removing the red blood cells by ACK lysing, the cells were stained with a panel of antibodies conjugated with either FITC or PE and analyzed on FACSCalibur with CellQuest Sorfware. The data shown in the figure are averages of triplicates. ${ }^{*} \mathrm{p}<0.05 ;{ }^{* *} \mathrm{p}<0.01$ and ${ }^{* * * *} \mathrm{p}<0.001$

excess water before being placed in coolers for fermentation at temperatures ranging from 24 to $30^{\circ} \mathrm{C}$ and humidity between $45-55 \%$. The fermentation process was terminated at 14 days. The resulting fermentation generated liquid exudate from the fruit was then collected in plastic bottles and frozen for storage prior to its use in the experiments described herein. Before injection, the liquid fermented Noni exudate (fNE) was thawed and adjusted to $\mathrm{pH}$ 7.0-7.4 with 50\% $\mathrm{NaOH}$.

CD marker profiles. C57BL/6J mice were intraperitoneally (i.p.) injected with three doses of fNE (500 $\mu \mathrm{l} /$ mouse/day) or three doses of PBS (500 $\mu 1 /$ mouse/day). One day after the injections, the mice were sacrificed and cells were collected from peripheral blood, spleen and peritoneum. After lysing the red blood cells with ACK solution, the cells were stained with a panel of antibodies (CD3, CD4, CD8, CD19, CD25, B220, CD11c, CD14, Gr-1 and NK1.1 from BD Biosciences) labeled with either FITC or PE and analyzed on a FACSCalibur using CellQuest software.

Tumor studies. In the preventive study, C57BL/6J (8 mice/ group), nude (4 mice/group) and beige mice (4 mice/group)

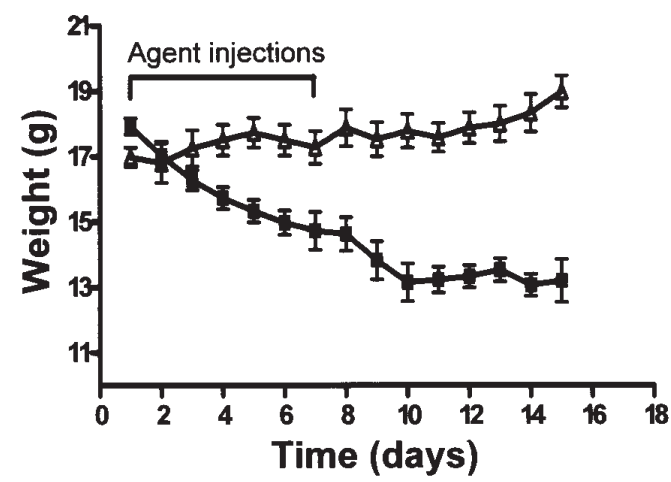

Figure 2. Growth rate of mice treated with PBS (triangles) or Noni (squares). C57BL/6J mice at age of 8 weeks were i.p. injected with seven doses of PBS $(500 \mu 1 /$ mouse/day) or seven doses of fNE $(500 \mu 1$ / mouse/day). The weight of the mice were measured every three days and graphed as an average in the figure.

were i.p. injected with three doses of fNE $(500 \mu 1 /$ mouse/day), three doses of LPS (0.125 $\mu \mathrm{g}$ /mouse/day) or PBS, respectively. On the fourth day, the mice were i.p. injected with S180 tumor cells or Lewis lung carcinoma LL/2 cells $\left(5 \times 10^{5}\right.$ cells/mouse). In the treatment study, S180 tumor cells were i.p. injected first and, a day later, the mice $(8 \mathrm{mice} /$ group for C57BL/6J, 4 mice/group for nude mice and beige mice) were i.p. injected with three doses of fNE (500 $\mu 1 /$ mouse/day), three doses of LPS $(0.125 \mu \mathrm{g} /$ mouse/day) or PBS. The survival of the mice was monitored. In order to determine whether there is an fNE-induced anti-tumor immunological memory, some of the mice that survived the first S180 inoculation were re-challenged with $1 \times 10^{6} \mathrm{~S} 180$ tumor cells two months after the first tumor challenge and $5 \times 10^{6} \mathrm{~S} 180$ tumor cells five months later. In order to determine whether the fNE-injection had any additional gross effects on the mice, the weight of a group of mice that received up to seven doses of fNE were recorded and compared to untreated mice.

fNE fractioning and tumor study. In order to determine what fraction of fNE contains the anti-tumor component, fNE was centrifuged at 10,000 rpm for $10 \mathrm{~min}$ and the supernatant was recovered and filtrated through a $0.2 \mu \mathrm{m}$ filter. The supernatant, fNEs, was then used in tumor study in a preventive setting. C57BL/6J mice (8/group) were i.p. injected with PBS, fNE, or fNEs for three days (500 $\mu 1 /$ day/mouse). On the fourth day, the mice were i.p. injected with S180 tumor cells $\left(5 \times 10^{5}\right.$ cells/ mouse). Survival of the mice was then monitored.

Statistical analysis. ANOVA and survival curve statistical analyses were performed using the built in software provided with GraphPad Prism ${ }^{\circledR} 4$.

\section{Results}

CD marker profiles. After fNE injection, the percentages of granulocytes and NK cells increased significantly in the three tissues: peripheral blood, spleen and peritoneum, while the percentage of $\mathrm{CD} 19^{+} \mathrm{B}$ cells decreased significantly in the three tissues (Fig. 1). The percentage of $\mathrm{CD}^{+} \mathrm{T}$ cells increased in the peritoneum, but decreased in the spleen, 
A
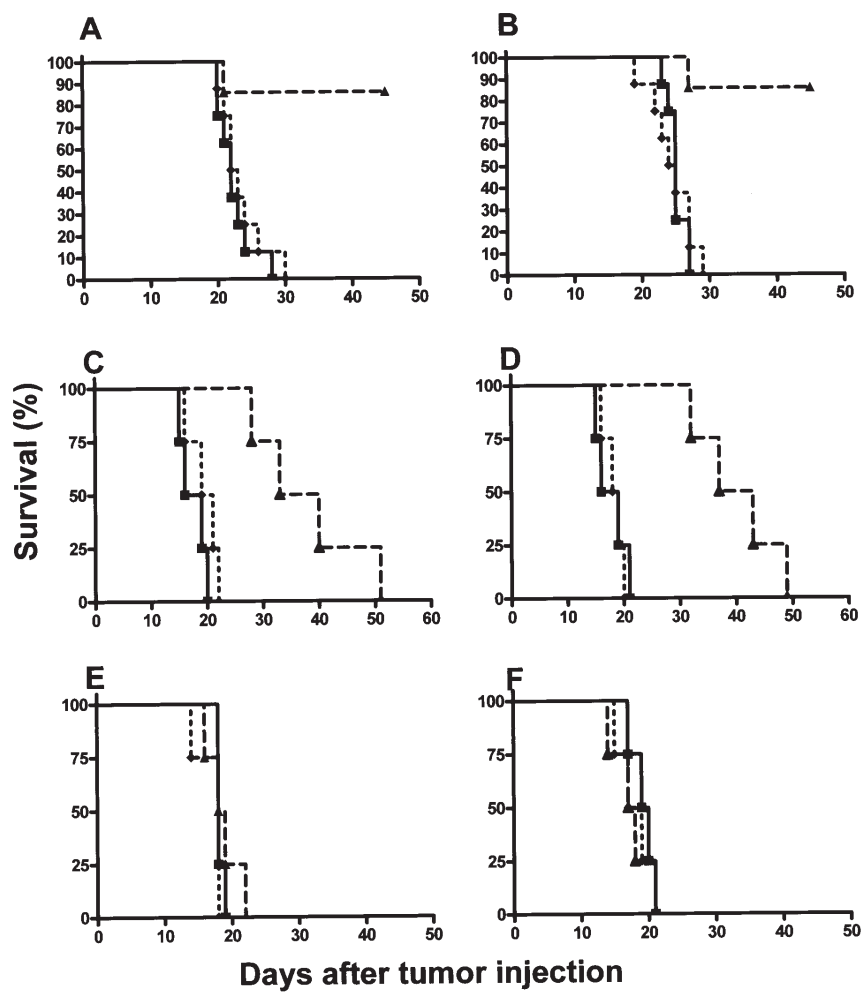

Figure 3. Anti-tumor effect of fNE injection in C57BL/6J mice (A and B), nude mice $(\mathrm{C}$ and $\mathrm{D})$, and beige mice $(\mathrm{E}$ and $\mathrm{F})$. In the preventive setting (A, $\mathrm{C}$ and $\mathrm{E})$, mice were given three doses of PBS (500 $\mu \mathrm{l} / \mathrm{mouse} / \mathrm{day}$, solid squares $)$, three doses of LPS $(0.125 \mu \mathrm{g} /$ mouse/day, dashed diamonds $)$, or three doses of fNE ( $500 \mu 1 /$ mouse/day, dashed triangles) by i.p. injections. A day later, the mice were i.p. challenged with $5 \times 10^{5} \mathrm{~S} 180$ tumor cells. In the treatment setting (B, D and F), tumor cells were injected first and a day later the mice were injected with three doses of PBS, three doses of LPS or three doses of fNE. The survival of the mice were monitored and graphed in the figure using GraphPad Prism 4 software.

while the percentages of $\mathrm{CD}^{+}$and $\mathrm{CD} 4^{+} \mathrm{T}$ cells decreased significantly in the spleen, but remained unchanged in the peritoneum and peripheral blood. fNE injection also significantly increased the peritoneal total leukocyte counts from an average of $1.47 \times 10^{6}$ in PBS injected mice to $15.78 \times 10^{6}$ cells in fNE-injected mice.

fNE-injected mice lost weight. In order to determine whether the injection of fNE interferes with the growth of mice, a group of C57BL/6J mice were given seven doses of fNE ( $500 \mu 1 /$ mouse/day) or PBS by i.p. injection. As Fig. 2 shows, the weight of the fNE-injected mice decreased as the agent was injected and the weight loss continued after the injections stopped. Three days after the end of injection, the animals stabilized and gradually recovered. Other than the weight loss, the animals appeared to be in excellent health.

fNE prevents and eradicates $S 180$ tumor in C57BL/6J mice. In the preventive study, mice were given three doses of $\mathrm{fNE}$ (500 $\mu \mathrm{l} / \mathrm{mouse} /$ day), three doses of LPS $(0.125 \mu \mathrm{g} / \mathrm{mouse} /$ day) or three doses of PBS (500 $\mu 1 /$ mouse/day) by i.p. injections. On the fourth day, all the mice were i.p. inoculated with $5 \times 10^{5} \mathrm{~S} 180$ tumor cells. In the treatment group, mice were each inoculated with $5 \times 10^{5} \mathrm{~S} 180$ tumor cells and the fNE, LPS or PBS treatment (fNE, $500 \mu 1$ /mouse/day; LPS,

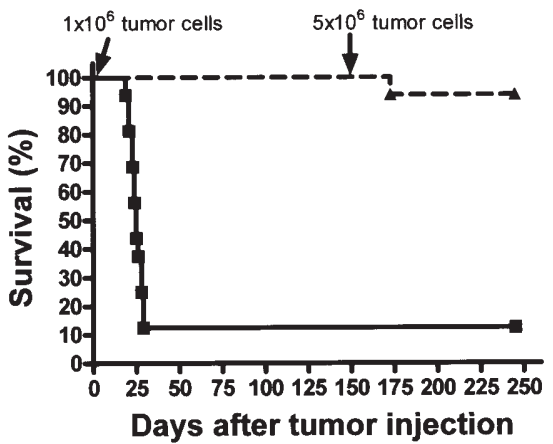

Figure 4. Tumor re-challenge. Sixteen C57BL/6J mice that received fNE treatment and tumor free were re-injected with $1 \times 10^{6}$ S180 tumor cells/mouse two months after the first tumor injection. Five months later, they were injected again with $5 \times 10^{6} \mathrm{~S} 180$ tumor cells/mouse (dashed triangles). Sixteen regular C57BL/6J mice were also injected with $1 \times 10^{6}$ S180 tumor cells (solid squares). The survival of the mice were monitored and graphed in the figure using GraphPad Prism 4 software.

$0.125 \mu \mathrm{g} / \mathrm{mouse} /$ day; or PBS, $500 \mu 1 /$ mouse/day) was started a day later. As indicated by Fig. $3 \mathrm{~A}$ and $\mathrm{B},>85 \%$ of the mice that received fNE, no matter whether in the preventive or treatment setting, were tumor-free one and a half months after tumor inoculation. Continued monitoring of these mice showed that they were tumor-free for their entire normal lifetime (data not shown), while all the mice in the control groups that received PBS or LPS developed ascites and died within 30 days of tumor inoculation.

LPS does not induce immune response against S180 tumors. There is a possibility that the anti-tumor activity of fNE is due to bacterial materials contained in it such as LPS. LPS content in fNE was determined and the result showed that the LPS level in $\mathrm{fNE}$ is $0.25 \mu \mathrm{g} / \mathrm{ml}$. Separate groups of mice including $\mathrm{C} 57 \mathrm{BL} / 6 \mathrm{~J}$, nude and beige mice were injected with LPS in an equivalent amount $(0.125 \mu \mathrm{g} / \mathrm{mouse} /$ day $)$. LPS at this dose did not show any anti-tumor activity either in the preventive setting or in the treatment setting (Fig. 3).

fNE prolonged the survival of nude mice receiving $S 180$ tumor cells. A similar experiment to that described above was performed with nude mice. As Fig. 3C and D shows, in the preventive and the treatment setting, fNE injection prolonged the survival of the nude mice significantly. However, as opposed to the normal C57bL/6J mouse experiment, in which $>85 \%$ of the mice receiving fNE treatment were tumor-free, all the nude mice eventually developed ascites and died.

fNE failed to prevent or eradicate $S 180$ tumors in beige mice. In order to determine if NK cells play any role in S180 tumor cell rejection following fNE treatment, beige mice, which do not have functional NK cells, were used in this study. Again, in the preventive study, beige mice were given three doses of fNE (500 $\mu 1 /$ mouse/day) or PBS (500 $\mu 1 /$ mouse/day) by i.p. injections. On the fourth day, all the mice were each injected i.p. with $5 \times 10^{5} \mathrm{~S} 180$ tumor cells. In the treatment group, beige mice were each injected with $5 \times 10^{5} \mathrm{~S} 180$ tumor cells and the fNE or PBS treatment (fNE, $500 \mu 1 /$ mouse/day or PBS, $500 \mu 1 /$ mouse/day) was started a day later. All the beige 


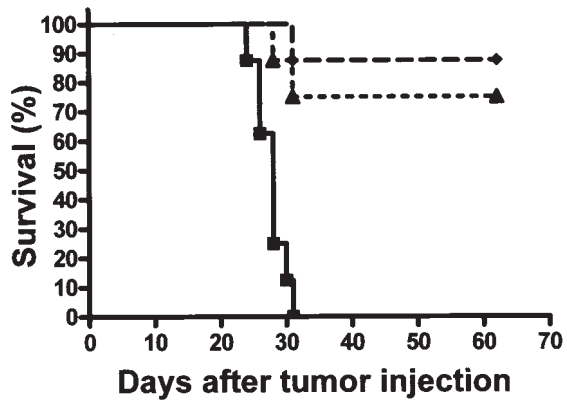

Figure 5. The supernatant of fNE contains the antitumor activity. fNE were centrifuged at 10,000 rpm. The supernatant was recovered, filtrated, and used in tumor study in a preventive setting. Mice were i.p. injected with three doses of PBS (500 $\mu 1 /$ mouse/day, solid squares), three doses of fNE (500 $\mu 1 /$ mouse/day, dashed diamonds), or three doses of fNEs (500 $\mu 1 /$ mouse/day, dashed triangles) by i.p. injections. A day later, the mice were i.p. challenged with $5 \times 10^{5} \mathrm{~S} 180$ tumor cells. The survival of the mice were monitored and graphed in the figure using GraphPad Prism 4 software.

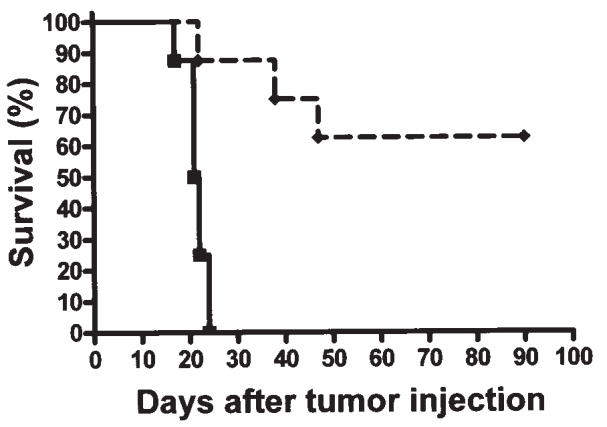

Figure 6. fNE injection protects C57BL/6J mice from Lewis lung tumor cell challenge. The antitumor activity of fNE was tested in a preventive setting. Mice were give three doses of PBS (500 $\mu 1 /$ mouse/day, solid squares) or three doses of fNE (500 $\mu 1 /$ mouse/day, dashed diamonds) by i.p. injections. A day later, the mice were i.p. challenged with $5 \times 10^{5} \mathrm{LL} / 2$ tumor cells. The survival of the mice were monitored and graphed in the figure using GraphPad Prism 4 software.

mice developed ascites and died 20 days after tumor inoculation (Fig. 3E and F).

Anti-tumor memory developed in C57BL/6J mice receiving $f N E$. Two months after the first tumor injection, C57BL/6J that received $\mathrm{fNE}$ and were tumor-free were re-challenged with $1 \times 10^{6}$ tumor cells/mouse. All the mice rejected the tumors. Five months later, the mice were re-challenged with $5 \times 10^{6}$ tumor cells. Fifteen of 16 mice $(93.75 \%)$ rejected the tumors (Fig. 4). In this study, we also discovered that 2 out of the 16 mice that received PBS as a control was tumor-free for its entire life.

Supernatant of fNE prevents $S 180$ tumor growth in mice. As a raw fermented material, fNE contains many components. In order to find out which component bears the anti-tumor activity, we first centrifuged and filtrated fNE through a $0.2 \mu \mathrm{m}$ filter. After heat inactivation, the supernatant of fNEs was used in tumor study. The result (Fig. 5) shows that the fNE is still effective in preventing S180 tumor growth in C57BL/6J mice.
fNE prevents LL/2 Lewis lung tumor in C57BL/6J mice. In order to determine whether the anti-tumor activity of fNE is restricted to S180 tumor cells, Lewis lung carcinoma tumor cell line LL/2, a highly tumorigenic tumor cell line was tested in the in vivo tumor study. Mice were given three doses of fNE ( $500 \mu \mathrm{l} / \mathrm{mouse} /$ day) or PBS (500 $\mu \mathrm{l} / \mathrm{mouse} /$ day) by i.p. injections. On the fourth day, all the mice were each i.p. inoculated with $5 \times 10^{5} \mathrm{LL} / 2$ tumor cells. As indicated by Fig. 6, $62 \%$ of the mice that received $\mathrm{fNE}$ survived LL/2 tumor cell challenge and lived tumor-free for $>3$ months, while mice that received PBS all died within 25 days after tumor cell injection.

\section{Discussion}

Several mechanisms have been proposed to understand how Noni manifests its anti-tumor effect, such as the inhibition of angiogenesis (4), prevention of carcinogen-DNA adduct formation (8), or activation of immune responses (7). In the present study we focused on the immune system, especially the innate immune system, due to the observation that fNE stimulated the infiltration of components of this system. A CD profile study (Fig. 1) demonstrated that the i.p. injection of fNE significantly increased the percentages of granulocytes and NK cells in peripheral blood, the peritoneum, and the spleen with more significant increases in the peritoneum and spleen $(p<0.001)$ than in peripheral blood $(p<0.05)$. Surprisingly, the fNE treatment significantly decreased the percentage of B lymphocyte in all the three settings and the percentage of $\mathrm{T}$ cells in the spleen. On the other hand, fNE increased the percentages of $\mathrm{CD} 8^{+} \mathrm{T}$ cells and $\mathrm{CD} 25^{+}$cells significantly in the peritoneum. Overall, NK cells were found to be the major responders of fNE treatment. As expected, the fNE treatment increased the total peritoneal leukocyte counts $>10$ fold.

Up to seven doses of fNE ( $500 \mu \mathrm{l} / \mathrm{mouse} /$ day) significantly decreased the body weight of the mice (Fig. 2) with mice starting to lose weight on the second day of fNE injection. When the fNE injections stopped, the mice kept losing weight for $\sim 3$ more days and then gradually stabilized and recovered. Besides the weight lost, the mice were generally healthy. Tumor studies as discussed below showed that three doses of fNE are enough to render the mice tumor resistant. Therefore, the weight lost may not represent a problem for fNE treatment.

Our data show that over $85 \%$ of the $\mathrm{C} 57 \mathrm{BL} / 6 \mathrm{~J}$ mice that received three doses of fNE completely rejected $\mathrm{S} 180$ tumor cells (Fig. 3A and B). This is in contrast to a previous study, in which Noni-ppt treatment only produced a cure rate of $25-45 \%$ (9). We believe that is due to the difference between Noni-ppt and fNE. Noni-ppt is a commercially produced and pasteurized agent, while the fNE we used in this study was the product of raw fermentation of the unprocessed Noni fruit. In our experiment using pasteurized Noni-ppt, only $\sim 50 \%$ of the mice treated were tumor-free (data not shown). Further study indicated that the anti-tumor activity remains in the supernatant of fNE (Fig. 5). Our current studies are focusing on characterization of the fNEs, hoping to discover one or a few natural anti-tumor products. In a different tumor model, our study demonstrated that fNE can also prevent Lewis lung carcinoma 
tumor cell (LL/2) growth in C57BL/6J mice (Fig. 6), although with a slightly lower tumor prevention rate $(62 \%)$, indicating that the anti-tumor activity is not restricted to S180 tumor cells.

The fact that LPS injection at the level of fNE $(0.25 \mu \mathrm{g} /$ ml) did not show any anti-tumor activity (Fig. 3) rules out the possibility that the anti-tumor activity of fNE is due to the contaminated bacterial materials. However, the anti-tumor activity may be due to pure Noni component(s) or a combination of Noni component(s) and the bacterial materials.

Furusawa et al reported that the anti-tumor activity of Noni-ppt was completely abolished by the concomitant administration of specific inhibitors of macrophages (2-chloroadenosine), T cells (cyclosporine) or nature killer cells (antiasialo GM1 antibody) (9). In our study, we chose two different systems to identify which cells play a major role in tumor rejection induced by fNE. In the nude mouse system, although all the mice eventually developed ascites, the fNE treatment significantly prolonged the survival of nude mice injected with S180 tumor cells (Fig. 3C and D). In contrast, the antitumor activity induced by fNE completely disappeared in beige mice lacking functional NK cells (Fig. 3E and F). These results indicate that the activation of the first line of defense of the innate immune system is absolutely necessary to kill the tumor cells. Without NK cells, as is the case in the beige mice, the mice were not be able to eradicate any tumor cells. However, just the innate immune system alone clearly is not enough for the mice to fully eradicate tumor challenge as shown by the studies in nude mice. Therefore, the collaboration of the two systems is very important for the animals to reject tumor cells. It would appear that fNE is a potent agent that can activate both aspects of the immune system to eradicate tumor cells.

\section{Acknowledgements}

We thank Eric Holle and his staff for the professional care of the mice used in this study and Lakendra Workman for her administrative contribution in this study. This study was supported in part by the GHS Oncology Foundation.

\section{References}

1. McClatchey W: From Polynesian healers to health food stores: changing perspectives of Morinda citrifolia (Rubiaceae). Integr Cancer Ther 1: 110-120, 2002.

2. Hirazumi A, Furusawa E, Chou SC and Kokama Y: Immunomodulation contributes to the anticancer activity of morinda citrifolia (noni) fruit juice. Proc West Pharmacol Soc 39: 7-9, 1996.

3. Hirazumi A, Furusawa E, Chou SC and Hokama Y: Anticancer activity of Morinda citrifolia (noni) on intraperitoneally implanted Lewis lung carcinoma in syngeneic mice. Proc West Pharmacol Soc 37: 145-146, 1994.

4. Hornick CA, Myers A, Sadowska-Krowicka H, Anthony CT and Woltering EA: Inhibition of angiogenic initiation and disruption of newly established human vascular networks by juice from Morinda citrifolia (noni). Angiogenesis 6: 143-149, 2003.

5. Kim IT, Park HJ, Nam JH, Park YM, Won JH, Choi J, Choe BK and Lee KT: In-vitro and in-vivo anti-inflammatory and antinociceptive effects of the methanol extract of the roots of Morinda officinalis. J Pharm Pharmacol 57: 607-615, 2005.

6. Liu G, Bode A, Ma WY, Sang S, Ho CT and Dong Z: Two novel glycosides from the fruit of Morinda citrifolia (noni) inhibit AP-1 transactivation and cell transformation in the mouse epidermal JB6 cell line. Cancer Res 61: 5749-5756, 2001.

7. Hirazumi A and Furusawa E: An immunomodulatory polysaccharide-rich substance from the fruit juice of Morinda citrifolia (noni) with antitumor activity. Phytother Res 13: 380-387, 1999.

8. Wang MY and Su C: Cancer preventive effect of Morinda citrifolia (noni). Ann NY Acad Sci 952: 161-168, 2001.

9. Furusawa E, Hirazumi A, Story S and Jensen J: Antitumor potential of a polysaccharide-rich substance from the fruit juice of Morinda citrifolia (Noni) on sarcoma 180 ascites tumor in mice. Phytother Res 17: 1158-1164, 2003.

10. Wong DK: Are immune responses pivotal to cancer patient's long term survival? Two clinical case-study reports on the effects of Morinda citrifolia (Noni). Hawaii Med J 63:1 82-184, 2004. 